Audiology

Neurotology
Audiol Neurotol 2009;14:411-422

DOI: $\underline{10.1159 / 000241898}$
Received: April 6, 2009

Accepted after revision: June 16, 2009

Published online: November 16, 2009

\title{
Development of a Microfluidics-Based Intracochlear Drug Delivery Device
}

\author{
William F. Sewell $^{\mathrm{a}}$ Jeffrey T. Borenstein ${ }^{\mathrm{b}} \quad$ Zhiqiang Chen $^{\mathrm{a}} \quad$ Jason Fiering ${ }^{\mathrm{b}}$ \\ Ophir Handzel $^{\mathrm{a}}$ Maria Holmboe ${ }^{\mathrm{b}}$ Ernest S. Kim ${ }^{\mathrm{b}}$ Sharon G. Kujawa ${ }^{\mathrm{a}}$ \\ Michael J. McKenna ${ }^{a}$ Mark M. Mescher ${ }^{b}$ Brian Murphy ${ }^{a, b}$ Erin E. Leary Swan ${ }^{b, c}$ \\ Marcello Peppi ${ }^{a}$ Sarah Tao ${ }^{b}$ \\ ${ }^{a}$ Massachusetts Eye and Ear Infirmary, Harvard Medical School, Boston, Mass., and \\ ${ }^{b}$ Draper Laboratory and ' Massachusetts Institute of Technology, Cambridge, Mass., USA
}

\section{Key Words}

Direct delivery $\cdot$ Drugs $\cdot$ Inner ear $\cdot$ Microfluidics

\begin{abstract}
Background: Direct delivery of drugs and other agents into the inner ear will be important for many emerging therapies, including the treatment of degenerative disorders and guiding regeneration. Methods: We have taken a microfluidics/ MEMS (MicroElectroMechanical Systems) technology approach to develop a fully implantable reciprocating innerear drug-delivery system capable of timed and sequenced delivery of agents directly into perilymph of the cochlea. Iterations of the device were tested in guinea pigs to determine the flow characteristics required for safe and effective delivery. For these tests, we used the glutamate receptor blocker DNQX, which alters auditory nerve responses but not cochlear distortion product otoacoustic emissions. $\boldsymbol{R e}$ sults: We have demonstrated safe and effective delivery of agents into the scala tympani. Equilibration of the drug in the basal turn occurs rapidly (within tens of minutes) and is dependent on reciprocating flow parameters. Conclusion: We have described a prototype system for the direct delivery of drugs to the inner ear that has the potential to be a fully implantable means for safe and effective treatment of hearing loss and other diseases.

Copyright $\odot 2009$ S. Karger AG, Basel
\end{abstract}

\section{Introduction}

Recent advances in the pharmacology and molecular biology of hearing have revealed new possibilities for preventing or minimizing hearing loss. Scientists and clinicians are making progress in understanding the molecular mechanisms associated with cochlear and auditory nerve degenerative processes [1] and in finding agents that can minimize degeneration and facilitate repair $[2,3]$. These insights offer the potential for development of novel and precise drug treatments. Moreover, the extraordinary progress that has been made in defining genes and protein activities offers hope for gene transfer and cell-based approaches to treat these diseases $[4,5]$. Therapies based on advances in cell and molecular biology will likely revolutionize the treatment of sensorineural hearing loss.

Emerging therapies will likely require direct delivery of drugs and other agents to the inner ear, and may require sequenced delivery of multiple and potentially unstable macromolecules for periods of months to years. It is likely that many newer agents will either have broad systemic effects and/or will be highly specific macromolecules needed to direct cell repair. In either case, direct delivery of the drugs to the inner ear will minimize side effects, enable access to cells of interest, and profoundly increase target specificity.

\section{KARGER}

() 2009 S. Karger AG, Basel

Fax +4161306 1234 E-Mail karger@karger.ch www.karger.com www.karger.com/aud
William F. Sewell

Department of Otolaryngology

Eaton Peabody Laboratory

243 Charles Street, Boston, MA 02114 (USA)

Tel. +1 617573 3156, Fax +1 617729 4408, E-Mail wfs@epl.meei.harvard.edu 
Emerging microsystem technologies present the opportunity to address many previously intractable bioengineering challenges associated with inner ear drug delivery. The extension of microfabrication methods drawn from the semiconductor industry has over the past $2 \mathrm{de}$ cades spawned MEMS (MicroElectroMechanical systems) devices capable of reproducing the functions of conventional sensors and actuators at a fraction of the size and cost $[6,7]$. These miniaturized devices with completely integrated electrical and mechanical functions have achieved commercial success in automotive, aerospace and consumer electronics applications, and medical device applications of MEMS technology are now emerging. The resulting miniaturization enables complete therapeutic and sensing systems to be integrated into devices small enough to be implanted in close proximity to the organ to be treated. In the case of drug delivery, complex automated dosing regimens can be pre-programmed into the system or even designed to respond to sensor input of physiological measurements in a closedloop feedback manner. Moreover, technologies have emerged that may allow controlled release of drugs in dried or lyophilized form from discrete compartments [8-10]. Methods that enable storage and release of solid phase compounds are also under development [11]. Such devices will offer powerful solutions because they will allow higher concentrations of drugs to be stored within the device and will also extend the long-term stability of therapeutic compounds.

We have developed a drug delivery system that will enable the timed and sequenced delivery of potentially unstable drugs into the perilymph of the inner ear. The initial design target for a prototype for use in humans is a completely implantable device capable of delivering agents for periods of months to years. Drugs will be stored in a concentrated stabilized form, and introduced into the perilymph under microprocessor control by a miniaturized low-power high-precision micropump. Ultimately, an integrated drug delivery device with sensors for monitoring flow, drug concentration and bioactivity will provide customized therapy based on individual responses to treatment.

The system uses a reciprocating fluid delivery regimen to deliver drugs through a single cannula acting as both inlet and outlet. The ability to recirculate inner ear fluid through the pump solves several problems. The recirculating fluid permits the reservoir to contain a highly concentrated solution, potentially allowing operation for years without a refill. This eliminates the possibility of microbial contamination during refill. In addition, the perilymph may travel through the catheter at a flow rate that is independent of that of drug delivery from the reservoir to the catheter. Thus, these 2 flow rates can be optimized separately. It is likely that frequent circulation of the perilymph will maintain patency in the catheter, as opposed to a slow one-way drug perfusion that might operate intermittently. Finally, because there is controlled supply of solvent, the drug storage could take any number of forms, such as microchip arrays, bioerodible polymers, or even hybrid combinations of these drug delivery methods.

Our approach to the development of this device has been to test the concepts first on the bench, and then in an animal model. For our animal model, we assessed the delivery of DNQX - a glutamate receptor antagonist - in the guinea pig cochlea. We exploited the tonotopic arrangement of the cochlea to provide biological assessment of the extent of delivery along the cochlear partition, with simultaneous assessment of damage to the inner ear. Herein, we summarize the successes and the challenges in the development of the device.

\section{Methods}

\section{Microfluidics/MEMS Technology}

Details of our fabrication methods and performance characteristics of the microfluidic components in our drug delivery systems have been previously published [12]. Several design features are critical to delivery system development: a capability for simple but robust connection methods of the various components into a working system, low leak rate and low permeability components and connections, and a design path which can ultimately lead to a delivery system with tightly integrated microcomponents that is suitable for human implantation. Our prototypes, in their current modular configurations, make use of friction-fit tube-overtube connections between components with added adhesive for improved leak resistance. Our microfabricated components are composed of laminated layers of polymer sheets, which are patterned and stacked to form necessary structures, e.g. compliant membranes and valves. Ports to these structures are achieved by in-plane lamination of either stainless steel or PEEK microtubing (typical outer diameter of $0.36 \mathrm{~mm}$ ). Two components can then be connected together by a single piece of thick-walled compliant tubing (e.g. silicone, Tygon ${ }^{\circledR}$ ) with a slightly undersized inner diameter that forms a tight seal over the components' rigid tube ports.

Two techniques have been developed to reduce gas and aqueous vapor transport, which result in fluid loss and bubble formation in the components themselves. Thin film metal encapsulation (e.g. $0.5-\mu m$-thick gold) significantly reduces vapor transport through the polymer materials composing our microfluidic components. The improvement in permeability must be traded off against possible adverse affects on other functional characteristics of the components. For example, thick metal encapsulation 
of a membrane will reduce the desired compliance. A second technique incorporates a capping method that creates a sealed vapor chamber over those portions of the component that have high permeability to gas and/or vapor (e.g. a thin compliant membrane). The materials composing this cap (typically glass or metal) are intrinsically of very low permeability. Fluid loss through the enclosed membrane is transient, stopping when the vapor cavity reaches a saturated vapor state. Similarly, gas transport from the cavity into the fluidic circuit stops when the partial pressure of gas in the cap equilibrates with the dissolved gas pressure in the liquid on the other side of the membrane.

Most of these components were fabricated by numerically controlled routing of sheets of Kapton polyimide or R/flex 1000 (Rogers) Kapton coated with thermoset adhesive using end mills of 0.008 - to 0.015-inch diameter in a T-Tech Quick Circuit 7000 precision X-Y table router. 3-D structures were then formed by laminating stacks of these machined sheets at $175^{\circ} \mathrm{C}$ and $2 \mathrm{MPa}$ pressure in pneumatic piston-type vacuum laminators, which we had previously developed specifically for this purpose. A typical device such as a valve will require the lamination of 5 separately machined layers, aligned in a fixture with pins. Rigid layers such as metal backing plates were laminated into the stack in the same way.

\section{Animals and Surgical Procedures}

Details of these procedures have been published [13]. Briefly, adult albino guinea pigs were anesthetized with a combination of pentobarbital sodium (Nembutal; $25 \mathrm{mg} / \mathrm{kg}$ i.m.), fentanyl $(0.2$ $\mathrm{mg} / \mathrm{kg}$ i.m.), and droperidol (10 $\mathrm{mg} / \mathrm{kg}$ i.m.) with booster doses given as needed. The tympanic bulla was exposed via a retroauricular incision and an opening on the bulla was created with a sharp blade to visualize the cochlear basal turn and the round window membrane. We placed the cochleostomy in the scala tympani near the round window membrane, drilling a hole approximately $175 \mu \mathrm{m}$ in diameter to fit the insertion of fused silica microcannula ( $145 \mu \mathrm{m}$ o.d., $75 \mu \mathrm{m}$ i.d.). The fused silica injection tubing was then inserted into the cochleostomy and sealed with dental cement. The compound action potentials (CAPs) were monitored near the round window niche. All procedures were conducted with the approval of the Animal Care and Use Committee of the Massachusetts Eye and Ear Infirmary.

\section{Drugs and Drug Delivery}

An artificial perilymph (AP) solution served as the control solution and vehicle for the drugs. Its composition was (in $\mathrm{mM}$ ): $\mathrm{NaCl}, 120 ; \mathrm{KCl}, 3.5 ; \mathrm{CaCl}_{2}$, 1.5; glucose, 5.5; HEPES, 20. The $\mathrm{pH}$ was adjusted with $\mathrm{NaOH}$ to 7.5 (total $\mathrm{Na}^{+}=130 \mathrm{mM}$ ). The glutamate receptor antagonist DNQX (6,7-dinitroquinoxaline-2,3-dione; $100 \mu \mathrm{M}, 300 \mu \mathrm{M}$ ) was dissolved in $\mathrm{AP}$ at desired concentrations and $\mathrm{pH}$-adjusted as needed. DNQX was first dissolved in a small amount of dimethyl sulfoxide (final concentration, 0.05\%), which does not, on its own, produce significant changes in cochlear responses [14].

\section{Stimulus Generation and Response Monitoring}

Sound stimuli were created and responses monitored using 16-bit A/D D/A boards (National Instruments) controlled in a LabVIEW environment by a PC workstation. Signals used to elicit CAPs and DPOAEs were delivered to the ear using the same custom coupler. The coupler accommodates transducers (Tucker
Davis EC1) and a Knowles EK3103 electret microphone to measure ear canal sound pressure via a probe tube concentric with the 2.54-mm (o.d.) sound delivery tube. Sensitivity vs. frequency calibration curves were generated for the monitoring and probe microphones, respectively, enabling conversion from voltage to sound pressure level (SPL; in $\mathrm{dB}$ re: $20 \mu \mathrm{Pa}$ ). The probe assembly was then placed at the animal's ear canal where 'in-animal' calibration sweeps were accomplished and used to determine the actual SPLs generated at the entrance to the bony canal. After calibration, the probe assembly remained in place for the duration of the experiment.

CAPs of the auditory nerve were elicited using tone-pip stimuli $\left(2.78-32.0 \mathrm{kHz}\right.$; $0.5-\mathrm{ms}$ duration, $0.5-\mathrm{ms}$ rise-fall; $\cos ^{2}$ onset envelope; $16 / \mathrm{s} ; 0-80 \mathrm{~dB}$ SPL in 5-dB steps). Activity detected locally by a silver wire electrode placed at the round window was amplified $(10000 \times)$, filtered $(300 \mathrm{~Hz}$ to $3 \mathrm{kHz}$ passband), and averaged (32 samples). CAP threshold was defined as the lowest stimulus level at which response peaks were clearly and reproducibly present.

When 2 primary tones $(\mathrm{f} 1, \mathrm{f} 2)$ are played simultaneously to the ear, acoustic distortion products of their interaction in the cochlea can be propagated back out, through the middle ear, and detected using a sensitive microphone placed in the external ear canal. The distortion product otoacoustic emission (DPOAE) corresponding to the frequency $2 \mathrm{fl}-\mathrm{f} 2$ is quite robust and is commonly used as a metric of cochlear function, and in particular outer hair cell function. These 2f1-f2 DPOAEs present in the canal sound pressure waveform were recorded as response amplitude vs. primary level functions $(\mathrm{L} 1=10-75 \mathrm{~dB}$ SPL; L2 = L1-10; primaries incremented together in 5 - $\mathrm{dB}$ steps) spanning the frequency range $\mathrm{f} 2=2.78-32.0 \mathrm{kHz}$ ( $\mathrm{f} 2 / \mathrm{f} 1=1.2$ ). Ear canal sound pressure was amplified, digitally sampled, averaged (25 discrete spectra at each frequency-level combination), and fast-Fourier transforms were computed from averaged pressures. DPOAE level at $2 \mathrm{f} 1-\mathrm{f} 2$ and surrounding noise floor values $( \pm 50 \mathrm{~Hz}$ of $2 \mathrm{f} 1-\mathrm{f} 2)$ were extracted. Iso-response contours (L2 levels required to generate a DPOAE amplitude criterion of 0 SPL) were constructed from amplitude vs. sound level data. Responses were measured at baseline (after bulla opening) and again following cochleostomy and silica tubing insertion, and at approx. 20- to 40-min intervals during perfusion of pre-drug, drug, and post-drug AP solutions.

\section{Results}

\section{Developing a System to Allow Reciprocating Drug Delivery into the Cochlea}

A fundamental characteristic of our delivery system is the use of a reciprocating fluid delivery profile. This system ejects fluid out of, and withdraws it back into, a single cannula connected to a closed microfluidic circuit. With reciprocating delivery, there is less chance of occlusion, as any material drawn into the tube would subsequently be expelled as fluid was forced out in the next cycle. This also simplified chemical compatibility with perilymph, because other than a small amount of AP required for 


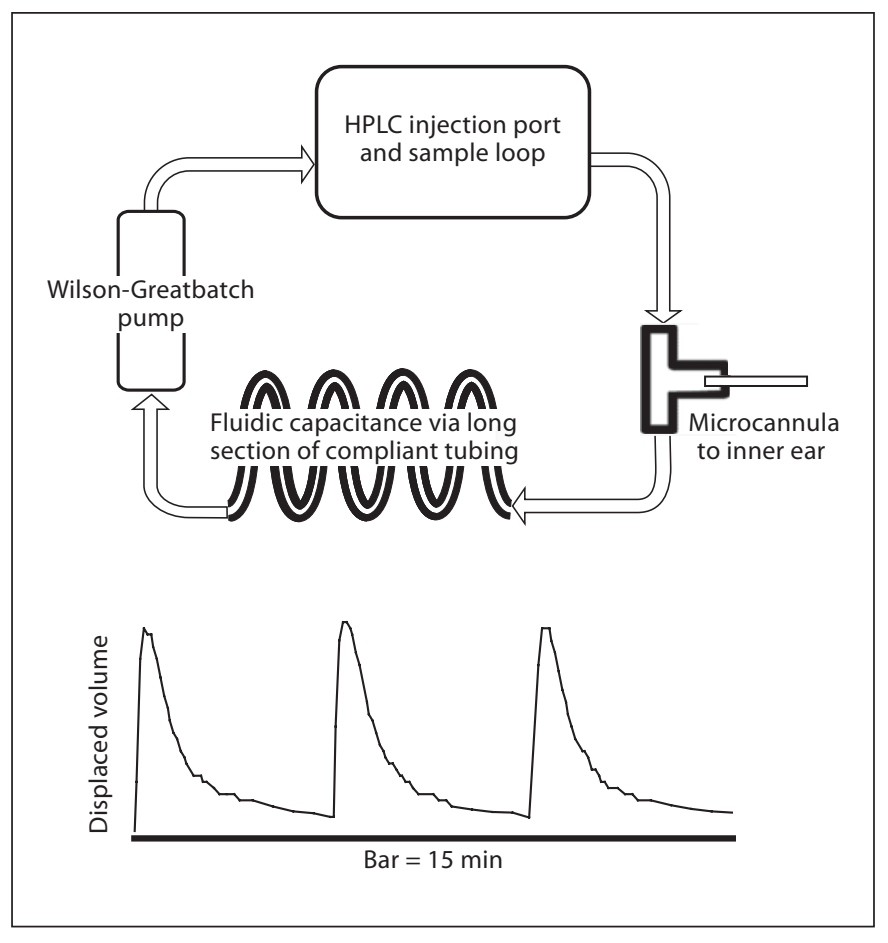

Fig. 1. Schematic representation of the Generation 1 device. We have previously published a thorough description of this device [13]. The device created a reciprocating delivery flow by recirculating perilymph through the system. A fluidic capacitance produced by a long length of compliant tubing produced a delay, measured at the manifold containing the ear cannula, between the positive pressure at the output of the pump and the negative pressure at the input. Placement of a high resistance between the manifold to the microcannula and the capacitor directed fluid out of the microcannula during the initial pulse. Thus, fluid was expelled through the cannula for a short period of time before being withdrawn back into the system.

priming, natural perilymph would ultimately serve as the vehicle for drug delivery. We have now produced 3 generations of devices built around the fundamental principle of reciprocating delivery, with each succeeding device providing additional flow control and a reduction in problems.

Our systems repeatedly inject a small volume ( 0.3 to a few $\mu \mathrm{l}$ ) of liquid into the cochlea over a short (few seconds) time, and then withdraw the liquid over a period of minutes. The systems produce no net flow of fluid into the cochlea because preliminary experiments demonstrated that the guinea pig inner ear cannot accommodate significant (more than $5 \mu \mathrm{l} / \mathrm{h}$ ) addition of fluid without an elevation in thresholds to acoustic stimulation.

The first-generation device was designed to provide proof of concept for reciprocating delivery using a recir- culating system (fig. 1). Analysis and testing of this device has been described [13]. The basic principle of the recirculating system is that a fluid pulse is ejected through the discharge capillary (e.g. a microcannula placed in the ear) and slowly drawn back into the recirculating loop. The system has the following characteristics: (1) the pump generates discrete volume displacements over short periods of time when in the on state (e.g. $0.5 \mu l$ over $30 \mathrm{~ms}$ ), and blocks flow in the reverse direction when in the off state; (2) the tubing and components of the pump-outlet portion of the loop and the outlet cannula have a combined fluidic compliance and flow resistance that result in a charging time constant on the order of seconds; (3) the tubing and components on the pump inlet side of the loop have a combined compliance and resistance resulting in a charging time constant on the order of minutes. The system operates in the following way: during excitation the pump displaces a net volume (e.g. in microliters) from its inlet to outlet, and thus charges the outlet to a high positive pressure and the inlet to a negative (gauge) pressure. Because of the designed difference in charging time constants of the loop, the pressured pump outlet is forced to quickly discharge out the cannula, rather than back into the pump-inlet side of the loop. The pump-inlet side of the loop slowly recharges, pulling fluid primarily from the cannula. Consequently fluid is ejected over a few seconds and then returns to the loop slowly over a period of minutes. The cycle may be repeated at any interval required to achieve the desired fluid delivery rate and circulation rate of perilymph. Multiple pulses may be applied in rapid succession to increase the pulse volume as long as the total pumping cycle time is less than the charging time constant. The result is a system capable of safely accommodating the pulse volumes from the pump, restricting the flow into and out of the ear, and producing no net average flow into the cochlea. Net delivery of dissolved compound is achieved through dilution occurring continuously as the solution is pumped into and back out of the cochlea.

The second-generation device (fig. 2) had 2 major changes. First, membrane-based microfluidic capacitors were fabricated to achieve relatively high compliance relative to component volume - an important step in miniaturization of the system. Second, the device was constructed using methods and materials that minimized gas and vapor transport through permeable materials. Long segments of flexible tubing can provide sufficient compliance to allow short-term operation of the recirculating loop. However, over long time periods, loss of fluid from the loop and subsequent entrainment of air bubbles 


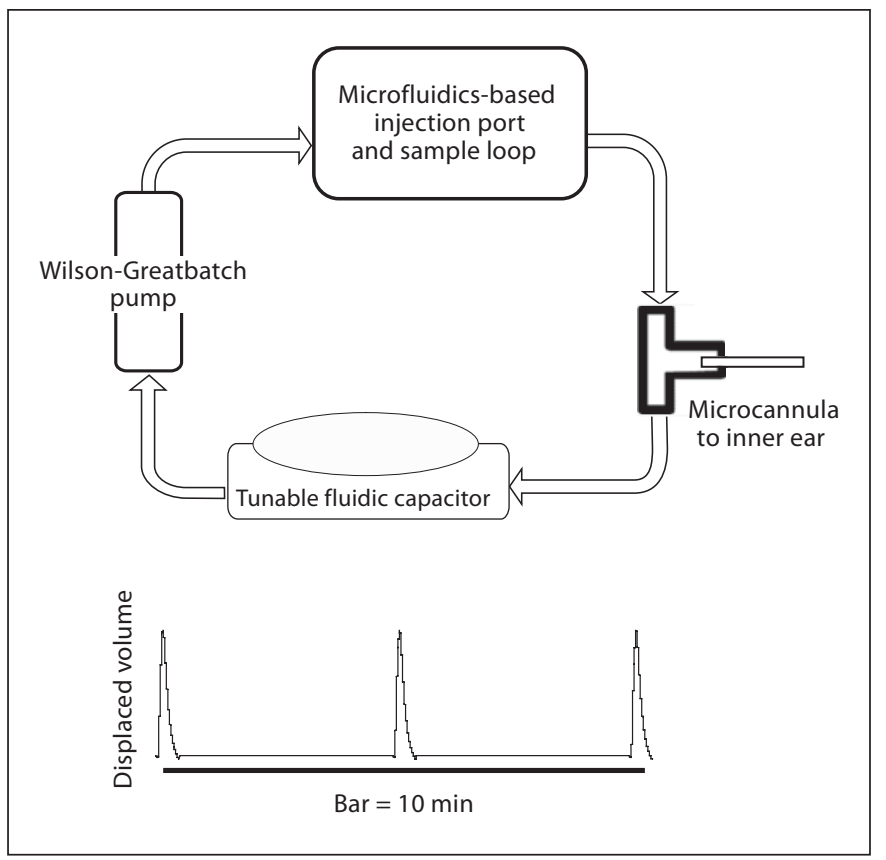

Fig. 2. Schematic representation of the Generation 2 device. The development of a tunable fluidics capacitor to replace the long length of tubing allowed better specification of the flow characteristics out of the microcannula. For this device, we also developed a microfluidics-based injection port and miniaturized the electronic control to allow the animal to wear the device for periods of days to weeks.

prevent reliable operation. The bubbles exhibit high compliance, and if they grow over time, they cause variable and unplanned compliances in the loop, and thus adversely alter the delivery profiles.

The third-generation device (fig. 3) represents a significant conceptual simplification of the reciprocating delivery system. In this version, the injection and withdrawal of fluid is controlled by a plunger, potentially providing much greater control over fluid delivery profiles without the need for a pump to prevent backflow when not excited. In addition, this system does not necessarily require controlled compliances to be designed into the system. The output is determined instead by the controlled displacement of the membrane actuator. For example, to generate a flow profile similar to that of the recirculating loop system described above, the piston actuator would apply a large force to the membrane over a few seconds to infuse fluid into the ear, and then reduce its force slowly over several minutes to permit the membrane to slowly return to its normal position while slowly refilling and thus withdrawing fluid from the ear.

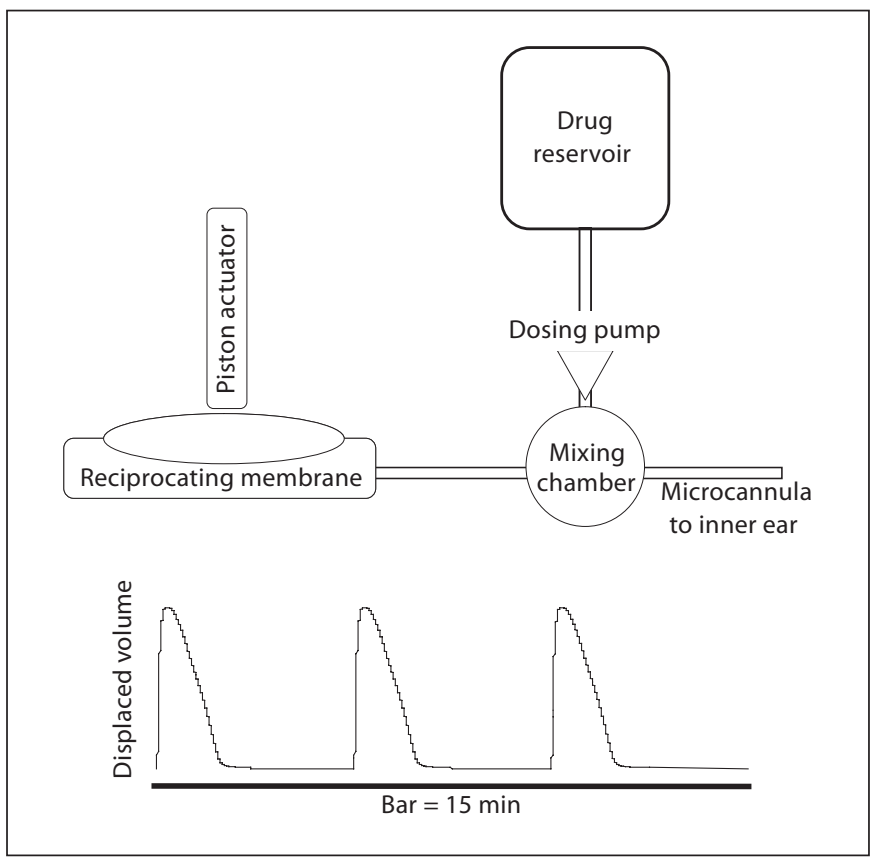

Fig. 3. Schematic representation of the Generation 3 device. In this device, the reciprocating delivery is achieved by a 'push-pull' fluid flow. In the initial benchtop experiments described in this paper, a syringe pump (Harvard Apparatus PHD2000) was substituted for the reciprocating membrane and piston actuator.

\section{Characterizing Fluid and Drug Delivery Parameters in Benchtop Versions of the Three Systems}

The recirculating systems (Generation 1 and 2 devices) were characterized by construction of a lumped electrical circuit analog, which allowed simulation of the flow and pressure characteristics using MicroCap software (Spectrum Software).

Simulation and measurement verified that the physiological pressures in the cochlea are not sufficient to measurably alter the discharge volume. The discharge capillary was connected to a static pressure head of $20 \mathrm{~cm}$ of water and the steady state motion of displacement of a meniscus was monitored and found to be unchanged. Static pressure in the perilymphatic compartment of the guinea pig is around $2.6 \mathrm{~cm}$ of water [15].

The second-generation recirculating device was created based upon the models for fluid delivery described here. We created microfluidic capacitors of polyimide laminates. The polyimide capacitors provided more reliable control of flow, and could be coated with materials that are impermeable to air to prevent the formation of air bubbles.

Audiol Neurotol 2009;14:411-422 


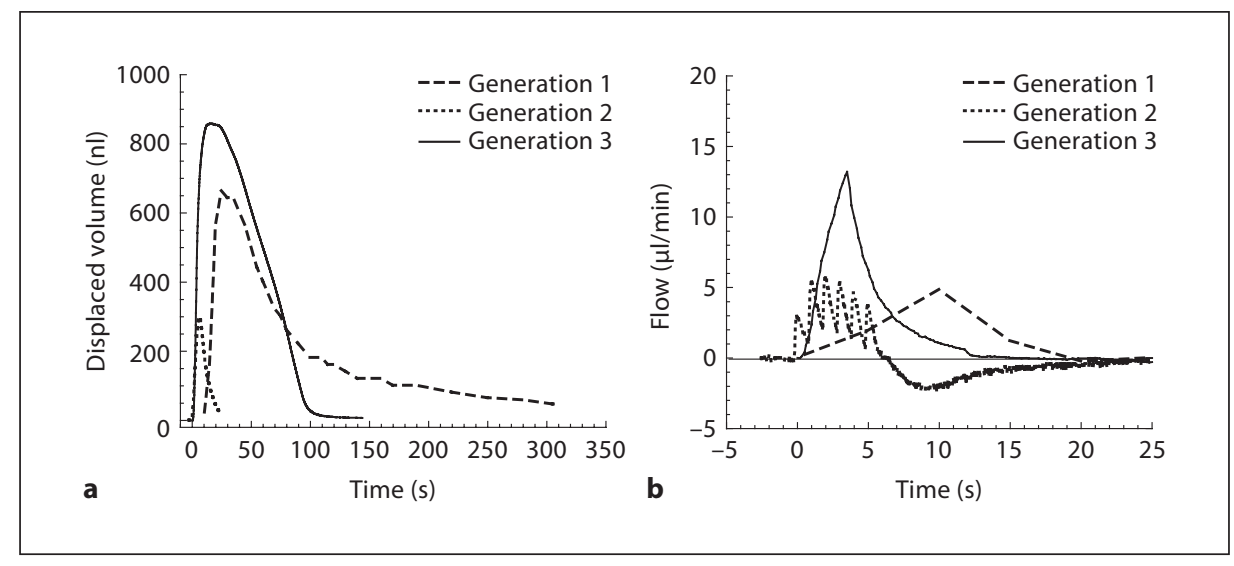

Fig. 4. Displaced fluid volume (a) and flow (b) as a function of time for each of the 3 devices. Displaced volume represents the volume of fluid injected into and then withdrawn from the inner ear. A positive flow indicates flow from the device to the cochlea and a negative flow is the return from the cochlea to the device. All 3 devices have both positive and negative flow components, though only the generation 2 device entered the negative flow mode during the time indicated in $\mathbf{b}(25 \mathrm{~s})$. In both the generation 1 and generation 2 devices, volume is displaced in each cycle by a closely spaced series of pump pulses. The capacitance properties of the generation 1 system integrate those closely spaced pump pulses more smoothly than the generation 2 device. In the generation 3 device, inflow and outflow are mediated by a positive and negative volume displacement via a 'push-pull' pump.
The third-generation (push-pull) device was conceptually far simpler, similar to control by a syringe pump, where the fluidic motion is described as a simple infusion and withdrawal controlled by displacement and withdrawal of a flexible membrane. A comparison of the fluid delivery characteristics of the 3 devices in figure 4 demonstrates the similarities (time course of fluid ejection and peak flow rates) and differences (rate of change of flow rates and ejection volumes) for the 3 iterations.

\section{Drug Delivery on the Bench}

An experiment was implemented for benchtop measurement of the system during multiple pump cycles. The goal was to measure over time the transport of a compound from the pump loop to an external reservoir, and thereby to obtain an effective infusion rate which incorporated both the diffusion and flow mechanisms occurring in the system. For drug injection in the guinea pig experiments, flow from the pump to the manifold was diverted into a sample loop to allow injection of the drug for a discrete period of time $(70 \mathrm{~min})$ followed by a period of wash. Performing a similar procedure with fluorescein and measuring concentration in the capillary tube indicated that concentrations of fluorescein during the injection period reached $80 \%$ of that in the sample loop in the first $0.5 \mathrm{~mm}$, and $50 \%$ at $1.5-2 \mathrm{~mm}$, during the period that fluorescein was present at the manifold.

\section{Delivery Systems in the Guinea Pig Showing}

Improvements in Drug Delivery to the Cochlea

The guinea pig has served for decades as the primary animal model for pharmacological work, and techniques for successful perfusion of the inner ear have been well studied. A cochleostomy can be made and AP perfused in the guinea pig with very little hearing loss [13].

Assessment of the distribution of the delivered drug along the length of the cochlea took advantage of the very well-characterized tonotopic organization of the cochlea, in which responses to high-frequency tones arise from the base low-frequency tones from the apex of the coiled tube, with a spatial relationship of about $3 \mathrm{~mm}$ per octave [16]. Two indicants of cochlear sensitivity and function were monitored: the outer-hair-cell-generated cochlear DPOAE, an acoustic signal measured in the external canal in response to bitonal stimulation, and CAP, an electrical signal evoked through synchronous activation of auditory nerve fibers by tone pips of varying frequency. An ideal drug for analyzing the distribution of agents throughout the cochlea is DNQX, which blocks glutamate receptors to prevent the generation of the CAP. Because it blocks at the synapse, the effects of the drug correlate to penetration of the drug throughout the cochlea. As it has no known action on outer hair cells, which generate the DPOAEs, monitoring those responses serves as an internal control for possible nonspecific/damaging effects of the fluid delivery, per se. 
As expected, the specific glutamatergic antagonist DNQX altered the CAP, but not the outer-hair-cell-generated DPOAEs. Drug effects on CAP were sensitive to stimulus frequency, consistent with the expected distribution of the drug along the length of the cochlea. Responses to high-frequency stimuli were altered first and most, followed progressively by involvement of responses to mid- and then lower-frequency stimuli. The frequency dependence is illustrated in figure 5 , where we show, for each of the 3 generations of the device, the thresholds during perfusion with $300 \mu \mathrm{M}$ DNQX plotted as a function of tone-pip frequency. The largest changes are at the basal (higher) frequencies. Though effects are larger with each successive version of the device, the penetrations of the drug into the apical regions of the cochlea were not very different. Figure 6 illustrates the time course of the effects of DNQX achieved with each of the three devices.

While improvements in the delivery device have increased the speed of drug effects and effectiveness in the basal portion of the cochlea, they have not improved penetration towards the apical region. One hypothesis, discussed below, is that protein binding of the drug may play a significant role in limiting the rate at which the drug migrates apically.

\section{Self-Contained Miniaturized System Worn on the}

\section{Head of the Guinea Pig}

Tethering the benchtop system to the guinea pig was associated with numerous challenges during chronic studies. The guinea pigs tended to turn more in one direction than in the other, which rapidly led to twisting and kinking of the infusion line, with a consequent disruption of flow. Instead, we successfully adapted a headmounted pod, previously developed to house components of a vestibular implant [17], to hold the pump, associated fluidic components, and miniaturized electronics and power supply.

Figure 7 depicts the assembled complete device. The system dimensions were $5.5 \times 4.0 \times 3.8 \mathrm{~cm}$. With 2 batteries in parallel, the system weighed $48 \mathrm{~g}$. This mass appears to be near the upper limit tolerated by large $(>600$ g), adult guinea pigs. In practice a single battery provides power for 2 weeks or more, reducing the typical mass to $42 \mathrm{~g}$. Units of this type were used routinely to cycle the system during long-term in vitro performance studies. The assembled system was mounted to the animal for 30 days, and measurements of the reciprocating outlet flow confirmed that the miniaturized system reproduces the behavior of our previous bench system.

Intracochlear Drug Delivery Device

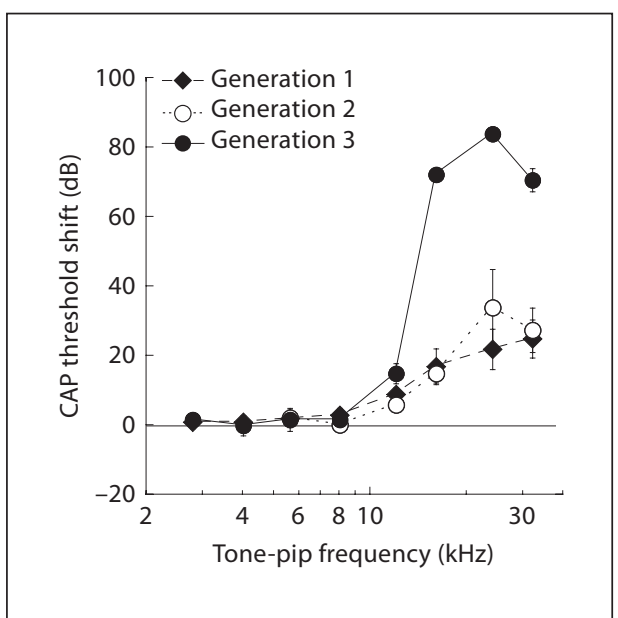

Fig. 5. CAP threshold shift, measured $1 \mathrm{~h}$ after DNQX infusion, is plotted as a function of tone-pip frequency. Cochlear regions near the base respond best to high-frequency tones. CAP threshold shift is related to the concentration of DNQX in each cochlear region. The 3 plots represent changes in CAP (means \pm SE) associated with the 3 devices. The flow and volume parameters for each device are indicated in figure 4 . For each device, flow and volume were optimized in a few initial experiments before settling on the parameters indicated in figure 4 .

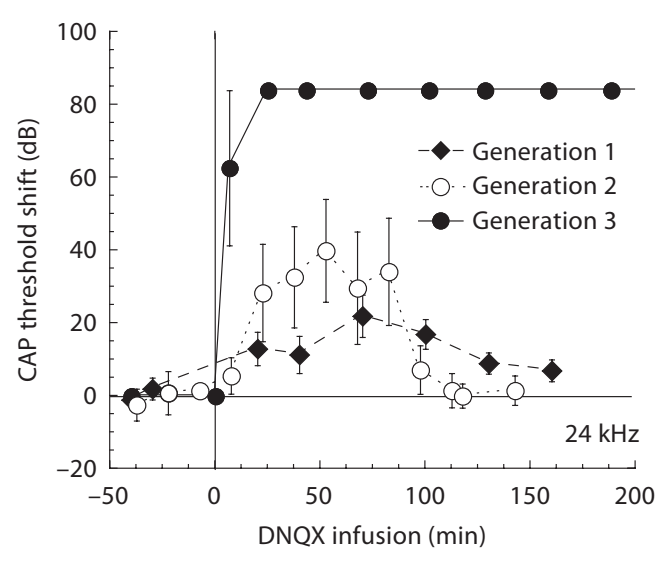

Fig. 6. Different reciprocating profiles of the 3 generations of our device produced differences in the rapidity and efficacy of drug effects. Data are means \pm SE.

\section{Bench Testing the Miniaturized System}

Measurements of displaced volume were made every few days over a period of 89 days to evaluate the stability of the displaced volume and the duration of the withdrawal cycle. The mean displaced volume was $1300 \mathrm{nl}$ per 


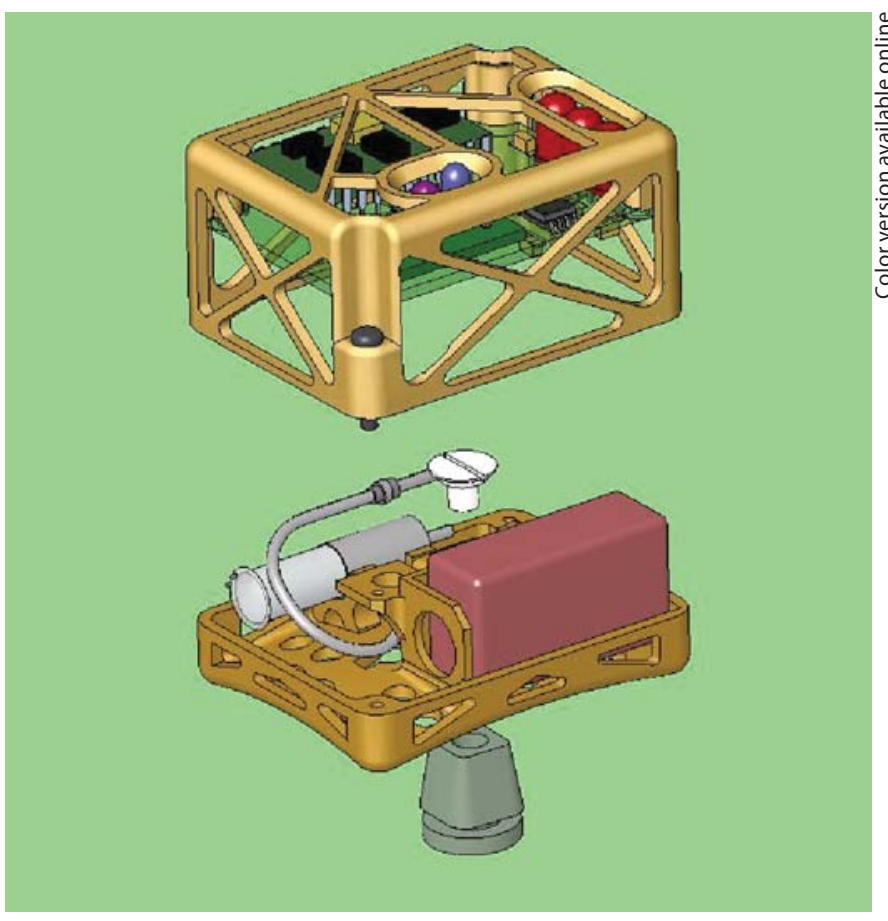

Fig. 7. Solidworks diagram of the miniaturized wearable device. The Wilson-Greatbatch pump, the microfluidics components, and the battery are placed on the lower level, while the upper level contains the microprocessor and the communications circuitry.

cycle with a standard deviation of $75 \mathrm{nl}$, while the mean time constant for the withdrawal was $6.9 \mathrm{~s}$ with a standard deviation of $0.7 \mathrm{~s}$. The maximum deviation from the mean in volume was $12.2 \%$ and in withdrawal time constant was $24 \%$. Greater than 40000 pump strokes and 10000 infuse/withdraw cycles were delivered in this study. After 89 days, the system was still operating normally, but was reconfigured for other purposes.

\section{Developing Components Resistant to Permeation}

We found that potential drift due to creep and fatigue of polymer membranes and tubing were a minor source of inconsistencies in pump output and could be neglected over the time periods studied. However, gas bubbles having volumes as low as $\sim 200 \mathrm{nl}$ could catastrophically disrupt the system's output if they occurred at critical locations. To address this liability, we instituted 4 critical procedures: (1) The system was thoroughly primed during the initial assembly, including the pump's internal mechanism. (2) All components and junctions were selected to minimize evaporative loss of water vapor and permeability to air. We sputter-coated a thin film of Au on the ca-

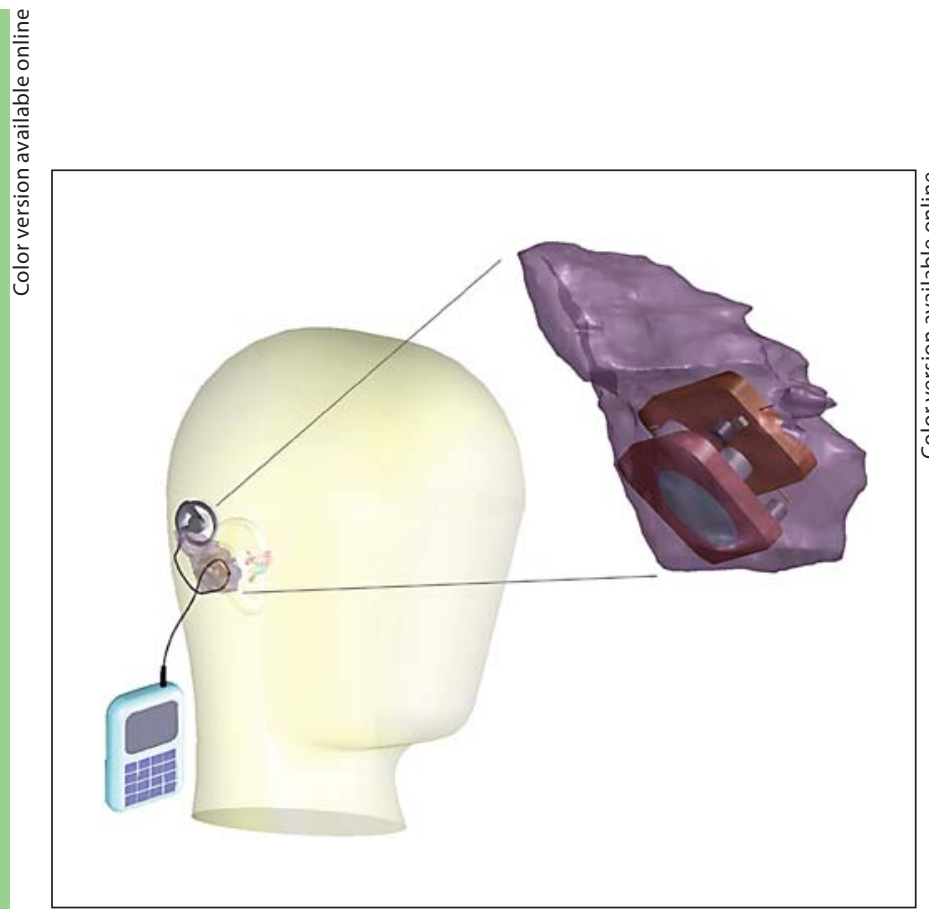

Fig. 8. Concept illustration of the device. The pump, fluid reservoir, and electronics are to be placed in space created in the mastoid. An external charger and control module will allow modification of the dose regimen and the drug delivery sequence.

pacitor polyimide membrane, and sealed the junctions between the resistor and pump with epoxy to prevent uptake of air during the momentary excursions below atmospheric pressure that these nodes experience. (3) The system was operated continuously. Interruptions of several hours for transport, surgery, or testing were benign, but if left static for several days, bubbles were commonly observed. (4) Components were designed to trap bubbles in preferred locations. For example, in the T-junction microchannels, a small bubble could alter resistance enough to disturb the output flow; hence, we controlled channel cross-sections to ensure that bubbles were swept through this component and did not exit through the cannula or remain lodged at an interface. The tendency of the fluidics capacitor to capture and retain bubbles before they entered the pump was advantageous, as this had little deleterious effect on system performance. To test the effectiveness of these methods, we measured the total system mass at intervals, and concluded that evaporation is compensated by water uptake from the reservoir when the outlet is immersed. 
Testing the System for Days to Weeks

Following implantation, system integrity was tested at periodic intervals by infusion of DNQX, and system safety was evaluated by monitoring cochlear sensitivity with ABRs and DPOAEs. In the early post-implantation period, we often experienced a transient hearing loss, which we attributed to middle ear problems since DPOAE thresholds were often elevated much more than $A B R$ thresholds, reflecting the impact of forward and reverse transmission through an abnormal middle ear. Ultimately, thresholds recover and are sensitive to subsequent drug challenges.

In the process of performing these chronic experiments, we have made numerous refinements in materials and placement of implanted components to maximize stability and minimize tissue injury. As components continue to be miniaturized, placement will be further optimized.

\section{Assessing Protein Composition of Perilymph to}

Address Biofouling and Pharmacokinetics

Because the device must function properly while interacting with intrinsic biological factors present in perilymph, we undertook a mass-spectrometry-based proteomics analysis of perilymph. Adsorption of proteins onto the surfaces of the device might lead to biofouling and changes in delivery profiles. Knowledge of protein composition of perilymph could help anticipate protein interactions with delivered agents and allow implants and drug delivery regimens to be tailored for optimal performance through the device's lifetime.

We adapted Salt et al.'s [18] perilymph collection procedures to the mouse to identify the most abundant proteins present in mouse perilymph and CSF. The mouse was chosen because its proteome is relatively complete and because of its increasing importance as a genetic model for human disease. The findings are summarized in Leary-Swan et al. [19] and only briefly summarized here. We found that while protein concentration in mouse perilymph is higher than that in cerebrospinal fluid (CSF), the composition is similar. The major drug-binding proteins, albumin, alpha-2-macroglobulin, and apolipoproteins, are found in abundance in perilymph. Albumin, the single protein found in the highest concentrations in perilymph, accounted for around $14 \%$ of the protein in perilymph. Other proteins in relatively high concentrations (5-6\%) included prostaglandin D synthase, 2 serine protease inhibitors (serpin ald and serpin a1a), and 2 apolipoproteins (apoA 2 and apoD). The major family of proteins in perilymph is the group of protease inhibitors, which represent over one-third of the total protein content in perilymph.

\section{Design Parameters and Physical Limitations of the Device in Human Implantation}

Implantation of the device in humans will require storage space and access to the middle or inner ears. The most acceptable space is the cavity created by a canalwall-up mastoidectomy. Detailed knowledge of the available space for implantation and pathways to access the middle and inner ears is necessary for the design of implants and successful implantation. Based on temporal bone CT scans of patients with normal middle ears, we prepared a $3 \mathrm{D}$ reconstruction of a virtual canal-wall-up mastoidectomy space to determine the maximal space that could be configured for implantation of the device [29]. The average volume, based on measurements from $32 \mathrm{CT}$ scans was $12.6 \mathrm{~cm}^{3}$ with standard deviation of 3.69 $\mathrm{cm}^{3}$, ranging from 7.97 to $23.25 \mathrm{~cm}^{3}$. Critical distances were measured directly from the model, and their averages were calculated: height $3.69 \mathrm{~cm}$, depth $2.43 \mathrm{~cm}$, length above the external auditory canal $4.45 \mathrm{~cm}$ and length posterior to the external auditory canal $3.16 \mathrm{~cm}$. These linear measurements did not correlate well with volume measurements. The shape of the models was variable to a significant extent, making the prediction of successful implantation for a given design based on linear and volumetric measurement unreliable. The measurements allowed us to configure a device shape (fig. 8) that should allow implantation in over $90 \%$ of patients. In addition, this method will allow preoperative assessment of mastoid cavity volume and dimensions and avoidance of surgery in the small percentage of individuals with inadequate mastoid space.

\section{Discussion}

\section{Reciprocating Delivery}

The use of a reciprocating fluid delivery system for drug delivery offers numerous advantages for organs with small fluid volumes. The ability to dissociate drug delivery from net fluid flow reduces the likelihood of damage to delicate structures, such as the organ of Corti, due to increased fluid volume or pressure, or to disruption of the normal fluid composition bathing the organ. While net fluid flow could be reduced using a separate inlet and outlet line, the possibility of fouling of the inlet (suction) line is high. The reciprocating system allows flushing of the cannula with positive pressure at every cycle. 
The design of the device incorporates recirculation of perilymph throughout the pump. An advantage of this feature is that one is assured compatibility of the fluid with perilymph. A potential disadvantage is that endogenous proteins in the perilymph have a greater opportunity to participate in adverse processes such as drug binding, drug metabolism, or generating protein films on the surface of the device that could lead to changes in the dynamics of fluid delivery.

Dosage control in the device is independent of the fluid being recirculated through the device. Dissociation of the drug delivery process from the fluid delivery process provides greater flexibility in dosage control and delivery timing. By varying the rate of delivery of drugs into the recirculating loop, it will be possible to control the concentration of the delivered drug. Timed delivery is possible as well, allowing increased or decreased delivery to allow adjustment of dosage in accordance with symptoms. Ultimately, it should be possible to store and deliver multiple drugs over varying times and sequences. Such a timed-sequenced delivery may be advantageous for developing therapeutic regimens for guiding inner ear regeneration.

The 3 generations of devices each had very different characteristics for flow and volume delivered over time. The efficacy of delivery did not correlate simply either to flow or to volume, which suggests a complex relationship between delivery parameters and efficacy of equilibration, at least within the basal turn region. However, the efficiency of delivery, as indicated by the rate and magnitude of changes associated with the injected DNQX, has increased with each successive iteration of the device. Distribution of the drug from the basal turn to upper turns may involve interactions with endogenous perilymph protein and tissue surrounding the scala tympani. Integrating and understanding the fluid dynamics with protein and tissue binding of the drug in the guinea pig, with anatomical and biochemical measurements in the human, will likely enhance the ability to distribute drugs throughout the cochlea in patients.

\section{Pharmacokinetic Considerations}

Direct delivery to the fluids of the inner ear is necessary because of the presence of a blood-labyrinth drug barrier, which is anatomically and functionally similar to the blood-brain barrier [20,21]. Direct delivery also has significant potential advantages for therapeutic application. Drugs are largely unaltered by metabolic changes that inevitably occur with other routes of administration, and have ready access to the sensory cells of the inner ear (the hair cells) and the synaptic regions of hair cells. Delivery directly to the inner ear can avoid undesirable systemic side effects that some drugs may produce. For most applications, getting drugs into the perilymph is most advantageous. Perilymph has access to most of the cells in the cochlea, the primary exception being those within the stria vascularis. While the scala media is a fluid space distinct from the perilymphatic space, all cells that form the epithelial border of the scala media are accessible via the perilymph.

The only alternative to direct administration of drugs into perilymph is via the round window membrane. The round window membrane is the Achilles' heel of the blood-cochlear barrier. This membrane is normally not exposed to other fluids of the body, and it has only 3 layers of cells. While 2 of these layers have tight junctions, there are numerous demonstrations that substances can pass through this membrane and that permeability can be induced [22]. It is possible to fill the middle ear space with fluid that can diffuse through the round window membrane into the perilymph space. However, there are a number of distinct disadvantages with this approach. One such drawback is the high degree of variability in the thickness of the round window membrane, the variable presence of an ancillary membrane covering the round window membrane, variable rates of loss of drug-containing fluid out of the middle ear into the eustachian tube, and the transient nature of the presence of the drug solution in the middle ear.

From a pharmacological perspective, direct administration into the perilymph is advantageous. There are several locations where drug solutions have been administered in animal models. The site of delivery can influence the accessibility of drugs to different elements of the inner ear. The most common route of entry is via a cochleostomy near the round window membrane. The primary advantage here is speed of access to the hair cells and afferent nerve fibers. Other approaches include: direct administration through the round window membrane and administration via the superior semicircular canal. Drugs can also be delivered via the CSF. In many animals, and especially in the guinea pig (which has been used extensively in pharmacological research), the cochlear aqueduct is large and patent, so fluid administered to the perilymph has a reasonable escape route. In most humans, the aqueduct offers limited continuity between the 2 fluid spaces.

We found a limited distribution of DNQX toward the apex of the cochlea following extended administration at the basal turn, which suggests that drug is eliminated or 
bound to protein and tissue at a significant rate. Our analysis of protein composition of perilymph confirmed and extended the earlier analysis of Thalmann et al. [23]. We found that while protein concentration in mouse perilymph is higher than that in CSF, the composition is similar. Many proteins found in perilymph may bind to the drug molecules making them unavailable to the target tissues. Albumin, $\alpha-1$ acid glycoprotein, prostaglandin D synthase, and lipoproteins may act in this way. Albumin tends to bind acidic ligands; $\alpha-1$ acid glycoprotein, found in relatively small quantities in perilymph, is the primary carrier for basic drugs; apolipoproteins and prostaglandin D synthase bind ligands with hydrophobic characteristics [24]. In addition, enzymes accounted for almost $15 \%$ of the protein in perilymph.

A concern with any implanted drug delivery device is the possibility of biofouling that can change the parameters of delivery over time. Extensive research on protein response to various materials is ongoing [25-27]. Because perilymph has a large amount of albumin, designs for implantable devices should incorporate biofouling considerations. Another known source of biofouling is highmolecular-weight kininogen, which is present in perilymph.

Drug solutions must be delivered at rates low enough to allow the cochlea to absorb the administered fluid to prevent damage to the cochlear elements. In an animal with a large cochlear aqueduct, the CSF space can serve as a sink for excess fluid that enters into the perilymph. However even with this 'relief valve', the cochlea is known to be sensitive to administered fluid. If drug solutions are delivered slowly enough to avoid damage to the cochlea, then uptake and removal of the drug becomes a potential problem. Drugs may have to be dissolved at relatively high concentrations to achieve therapeutic concentrations in the most apical regions of the cochlea.

If one is to use the cochlear aqueduct and fluid pathway to the CSF as a sink for fluid administered into the inner ear, it is important to carefully choose the inlet hole. For an inlet hole in the scala tympani near the round window, most of the applied fluid will likely flow immediately into the cochlear aqueduct and miss most of the structures of the cochlea. Thus, the inlet hole can be thought of as a point source from which diffusion must take place. One could maximize the amount of flow through the cochlea by administering fluid into the scala vestibuli, or perhaps the superior semicircular canal, which is relatively easy to access. However, the drug must then travel along the length of the scala vestibuli before it begins to enter the scala tympani and access the organ of Corti.

\section{General Considerations for Implantable Inner Ear Drug Delivery}

The device we are developing is well suited for inner ear drug delivery in many ways, particularly when considering the expected requirements for emerging therapies for hearing regeneration [28]. It will be of appropriate size and form to be implanted in the mastoid cavity near the cochlea. The device will be capable of storing one or more drugs in a concentrated form that can be diluted into perilymph, reducing the need for refills and the consequent potential for contamination. It will incorporate externally programmable control of dosing to enable adjustment of dose in response to exacerbation or remission of symptoms. It will enable programmable timed-sequenced dosing, which may be advantageous in guiding inner ear regeneration. The use of reciprocating fluid delivery through a single port is expected to reduce the likelihood of biofouling, and may reduce the potential for hearing damage by introducing the drug without net changes in fluid volumes within the inner ear. Before inner ear drug delivery can become a common treatment option for patients, many surgical and related issues must be addressed and resolved. Some are straightforward, e.g. placement of the device in the mastoid will use surgical approaches developed for cochlear implants the cannula might be placed into the ear through the round window with devices being developed for cochlear implants. Explantation and reimplantation of the device may present difficulties for preservation of residual hearing. Other challenges, such as eliminating routes of infection and preventing tissue ingrowth, may require new engineering or surgical methods. Ultimately, the development of new devices and approaches for intracochlear drug delivery, coupled with advances in understanding degeneration and regeneration in the inner ear will lead to new pharmacological therapies for deafness and other disorders.

\section{Acknowledgement}

This work was supported in part by grant number 5R01DC006848-03 from the NIH NIDCD. 


\section{References}

$\rightarrow 1$ Holley MC: Application of new biological approaches to stimulate sensory repair and protection. Br Med Bull 2002;63:157-169.

$\checkmark 2$ Ryan AF, Harris JP, Keithley EM: Immunemediated hearing loss: basic mechanisms and options for therapy. Acta Otolaryngol Suppl 2002;548:38-43.

3 Shinohara T, Bredberg G, Ulfendahl M, Pyykko I, Olivius NP, Kaksonen R, Lindstrom B, Altschuler R, Miller JM: Neurotrophic factor intervention restores auditory function in deafened animals. Pro Natl Acad Sci USA 2002;99:1657-1660.

4 Raphael Y: Cochlear pathology, sensory cell death and regeneration. Br Med Bull 2002 63:25-38.

$\checkmark 5$ Lalwani AK, Jero J, Mhatre AN: Developments in cochlear gene therapy. Adv Otorhinolaryngol 2002;61:28-33.

-6 Madou M, He K-Q: Exploitation of a novel artificial muscle for controlled drug delivery. Polym Mater Sci Eng 2000;83:495-497.

7 Kovacs GTA: Micromachined Transducers Sourcebook. New York, McGraw-Hill, 1998.

$>8$ Langer R: Drug delivery: drugs on target. Science 2001;293:58-59.

$\checkmark 9$ Santini JTJ, Cima MJ, Langer R: A controlled-release microchip. Nature 1999;397: 335-338.

10 Madou MJ: Fundamentals of micromachining. Boca Raton, CRC, 2002.

11 Mescher MJ, Dube C, Fiering J, Fyler D, Hansberry M, Kim E, Borenstein J, Bernstein J, Gragoudas E, Miller J: A programmable device for long-term transscleral drug delivery. Biomaterials Society Annual Meeting 2006.

12 Mescher MJ, Swan EEL, Fiering J, Holmboe M, Sewell WF, Kujawa SG, McKenna MJ, Borenstein JT: Fabrication methods and performance of low-permeability microfluidic components for a miniaturized wearable drug delivery system. IEEE JMEMS, in press.
13 Chen Z, Kujawa SG, McKenna MJ, Fiering JO, Mescher MJ, Borenstein JT, Swan EEL, Sewell WF: Inner ear drug delivery via a reciprocating perfusion system in the guinea pig. J Control Release 2005;110:1-19.

14 Littman T, Bobbin RP, Fallon M, Puel JL: The quinoxalinediones DNOX, CNOX and two related congeners suppress hair cell-to-auditory nerve transmission. Hear Res 1989;40: 45-53.

15 Wit HP, Thalen EO, Albers FW: Dynamics of inner ear pressure release, measured with a double-barreled micropipette in the guinea pig. Hear Res 1999;132:131-139.

16 Tsuji J, Liberman MC: Intracellular labeling of auditory nerve fibers in guinea pig: central and peripheral projections. J Comp Neurol 1997;381:188-202.

-17 Merfeld DM, Gong W, Morrissey J, Saginaw M, Haburcakova C, Lewis RF: Acclimation to chronic constant-rate peripheral stimulation provided by a vestibular prosthesis. IEEE Trans Biomed Eng 2006;53:23622372.

18 Salt AN, Hale SA, Plontke SK: Perilymph sampling from the cochlear apex: a reliable method to obtain higher purity perilymph samples from the scala tympani. J Neurosci Methods 2006;153:121-129.

19 Leary-Swan EE, Peppi M, Chen Z, Green KM, Evans JE, McKenna MJ, Mescher MJ, Kujawa SG, Sewell WF: Proteomics analysis of perilymph and cerebrospinal fluid in mouse. Laryngoscope 2009;119:953-958.

20 Inamura N, Salt AN: Permeability changes of the blood-labyrinth barrier measured in vivo during experimental treatments. Hear Res 1992;61:12-18.
-21 Juhn S, Rybak L: Labyrinthine barriers and cochlear homeostasis. Acta Otolaryngol 1981;91:529-534.

22 Schachern PA, Paparella MM, Duvall AJ 3rd: The normal chinchilla round window membrane. Arch Otolaryngol 1982;108:550-554.

23 Thalmann I, Kohut R, Ryu J, Comegys T, Senarita M, Thalmann R: Protein profile of human perilymph: in search of markers for the diagnosis of perilymph fistula and other inner ear disease. Otolaryngol Head Neck Surg 1994;111:273-280.

24 Goldstein A, Aronow L, Kalman S: Principles of Drug Action: The Basis of Pharmacology, ed 2. New York, John Wiley and Sons, 1974.

25 Sasaki DY, Cox JD, Follstaedt SC, Curry MS, Skirboll SK, Gourley PL: Glial cell adhesion and protein adsorption on SAM coated semiconductor and glass surfaces of a microfluidic structure. Proc SPIE 2001;4265:152163.

26 Richardson RRJ, Miller JA, Reichert WM: Polyimides as biomaterials: preliminary biocompatibility testing. Biomaterials 1993;14: 627-635.

27 Lim C, Slack S, Ufer S, Lindner E: Protein adsorption to planar electrochemical sensors and sensor materials. Pure Appl Chem 2004;76:753-764

28 Swan EE, Mescher MJ, Sewell WF, Tao SL, Borenstein JT: Inner ear drug delivery for auditory applications. Adv Drug Delivery Rev 2008;60:1583-1599.

29 Handzel O, Wang H, Fiering J, Borenstein JT, Mescher MJ, Swan EE, Murphy BA, Chen Z, Peppi M, Sewell WF, Kujawa SG, McKenna MJ: Mastoid cavity dimensions and shape: method of measurement and virtual fitting of implantable devices. Audiol Neurotol 2009;14:308-314. 